\title{
Comparing Spatial and Scenario Decomposition for Stochastic Hydrothermal Unit Commitment Problems
}

\author{
Murilo Reolon Scuzziato, Erlon Cristian Finardi, Antonio Frangioni
}

\begin{abstract}
Solving very-large-scale optimization problems frequently require to decompose them in smaller subproblems, that are iteratively solved to produce useful information. One such approach is the Lagrangian Relaxation (LR), a general technique that leads to many different decomposition schemes. The LR produces a lower bound of the objective function and useful information for heuristics aimed at constructing feasible primal solutions. In this paper, we compare the main LR strategies used so far for Stochastic Hydrothermal Unit Commitment problems, where uncertainty mainly concerns water availability in reservoirs and demand (weather conditions). The problem is customarily modeled as a two-stage mixed-integer optimization problem. We compare different decomposition strategies (unit and scenario schemes) in terms of quality of produced lower bound and running time. The schemes are assessed with various hydrothermal systems, considering different configuration of power plants, in terms of capacity and number of units.
\end{abstract}

Index Terms - Lagrangian Relaxation, Mixed-Integer Linear Programming, Hydrothermal Stochastic Unit Commitment.

\section{NOMENCLATURE}

\begin{tabular}{|c|c|}
\hline$p t$ & Vector of thermal power generation (MW). \\
\hline$u$ & Vector of commitment status of thermal plants. \\
\hline up & Vector of startup status of thermal plants. \\
\hline$u d$ & Vector of shutdown status of thermal plants \\
\hline$F$ & Vector of the thermal production cost $(\mathrm{R} \$)$. \\
\hline$p h$ & Vector of hydro power generation (MW). \\
\hline$v$ & Vector of volume in the reservoirs $\left(\mathrm{hm}^{3}\right)$. \\
\hline$d$ & $\begin{array}{l}\text { Vector of hydro outflow, i.e., sum of turbined } \\
\text { outflow and the spillage }\left(\mathrm{m}^{3} / \mathrm{s}\right) \text {. }\end{array}$ \\
\hline$s$ & Vector of spillage $\left(\mathrm{m}^{3} / \mathrm{s}\right)$ \\
\hline phg & $\begin{array}{l}\text { Vector of generation of a group of identical units } \\
\text { in the same hydro plant (MW). }\end{array}$ \\
\hline$q$ & $\begin{array}{l}\text { Vector of turbined outflow by a group of identical } \\
\text { units in the same hydro plant }\left(\mathrm{m}^{3} / \mathrm{s}\right) \text {. }\end{array}$ \\
\hline$z$ & $\begin{array}{l}\text { Vector of commitment status of the group of } \\
\text { units. }\end{array}$ \\
\hline$n$ & Index of nodes in the scenario tree $(n=1, \mathrm{~N})$. \\
\hline $\mathrm{N}$ & Number of nodes in the scenario tree. \\
\hline$p_{n}$ & Probability of node $n$. \\
\hline$f_{n}$ & $\begin{array}{l}\text { Operational cost function of node } n \text {, given by the } \\
\text { thermal generation and startup costs }(\mathrm{R} \$) \text {. }\end{array}$ \\
\hline I & Number of thermal plants, such that $i=1, \mathrm{I}$. \\
\hline $\mathrm{R}$ & Number of hydro plants, such that $r=1, \mathrm{R}$. \\
\hline $\mathbf{J}_{r}$ & Number of groups in the hydro $r$, with $j=1, \mathrm{~J}_{r}$. \\
\hline
\end{tabular}

This work received its financial support in part by CNPq.

M R. Scuzziato is with Departamento Acadêmico de Eletrotécnica, Instituto Federal de Santa Catarina (IFSC), Brazil (murilo.scuzziato@ifsc.edu.br). E. C. Finardi is with the Departamento de Engenharia Elétrica, Universidade Federal de Santa Catarina (UFSC), Brazil (erlon.finardi@ufsc.br). A. Frangioni is with Dipartimento di Informatica, Università di Pisa, Pisa, Italy (frangio@di.unipi.it).
$\Omega \quad$ Number of scenarios, such that $\omega=1, \Omega$.

$N(\omega) \quad$ Set of all the nodes of scenario $\omega$.

CA Number of cascades.

pta Vector of auxiliary variables for thermal power generation (MW).

pha Vector of auxiliary variables for hydropower generation (MW).

$v a \quad$ Vector of auxiliary variables for volumes in reservoirs $\left(\mathrm{hm}^{3}\right)$.

da Vector of auxiliary variables for hydro outflow $\left(\mathrm{m}^{3} / \mathrm{s}\right)$.

$\operatorname{Res}_{n} \quad$ Spinning reserve requirement for node $n$ (MW).

$\lambda p t$, $\gamma p t$ Dual variables vectors (Lagrange multipliers) $p t$ $(\mathrm{R} \$ / \mathrm{MW})$.

$\lambda p h, \quad$ Dual variables vectors related to $p h(\mathrm{R} \$ / \mathrm{MW})$.

$\gamma p h$

Ares Dual variables vectors related to spinning reserve constraint (R\$/MW).

$\lambda v, \gamma v \quad$ Dual variables vectors related to $v\left(\mathrm{R} \$ / \mathrm{hm}^{3}\right)$.

$\lambda d \quad$ Dual variables vectors related $d\left(\mathrm{R} \$ \cdot \mathrm{s} / \mathrm{m}^{3}\right)$.

$\Phi^{\mathrm{D}} \quad$ Set of subproblems a), one for each node.

$\Phi^{\mathrm{T}} \quad$ Set of subproblems b), one for each thermal plant.

$\Phi^{\mathrm{H}} \quad$ A single subproblem c) concerning all hydro plants.

$\Phi^{\mathrm{HC}} \quad$ Set of subproblems d).

$\Phi^{\mathrm{HA}} \quad$ Set of subproblems e).

$\Phi^{\mathrm{HE}} \quad$ Set of subproblems f).

$\Phi_{\omega}^{\mathrm{C}} \quad$ UC subproblem associated with scenario $\omega$.

$\Phi_{X} \quad$ Probability for method $X$.

fm Multiplying factor.

$\eta_{X}(p c)$ Performance metric for method $X$ to solve case $p c$.

$\eta^{*}(p c)$ Best performance metric found with whatever method.

$p c \quad$ A case of the problem, where $p$ belongs to a representative set of cases.

$f_{i}^{3} \quad$ Startup cost for thermal plant $i(\mathrm{R} \$)$.

$y_{r n} \quad$ Incremental inflow of hydro $r$ and node $n\left(\mathrm{~m}^{3} / \mathrm{s}\right)$.

$v_{r}^{\text {target }}$ Volume target for hydro $r$ at the end of horizon $\left(\mathrm{hm}^{3}\right)$.

fpgh Hydro production function

CH Number of hydro chains, such that $c h=1, \mathrm{CH}$.

$\alpha \quad$ Thermal operational cost coefficients.

$D_{b n} \quad$ Demand requirement at bus $b$ and node $n$ (MW).

$\Gamma \quad$ Power transfer distribution factor.

Other nomenclatures with superscript $\max$ or $\min$ represents the limits values for a specific variable vector.

\section{INTRODUCTION}

$\mathrm{M}$ OST real-world problems can be mathematically formulated using nonlinear functions and integer varia- 
bles, which leads to hard Mixed-Integer Nonlinear Problems (MINLP). In general, the constraints of these MINLPs have a high degree of structure, encouraging the use of techniques that decomposes them. One of the most applied such methods is the Lagrangian Decomposition or Lagrangian Relaxation (LR) [1], [2] in which the original problem is decomposed into several independent subproblems, possibly with different sizes and mathematical nature. For further basic information of LR, as well as its applications, see references [3], [4]. The LR is very flexible: several decomposition schemes can be developed for the same problem, with complex trade-offs between the computational cost and the quality of the results [1]. In this paper, we assess this flexibility applying the LR in electrical power systems based on real data. The main information produced by LR approaches are bounds on the optimal value of the problem and the corresponding Lagrangian multipliers, that have possible uses (e.g., to estimate prices of resources). However, they also provide valuable primal information, such as "convexified" primal solutions that can be used as the basis of heuristic approaches for producing feasible solutions [1], [5], and "convexifed" future value functions [6] that can also be used for the bidding problem [7]. The LR requires the solution of a convex nondifferentiable optimization problem, for which one of the most effective approaches is the Bundle Method (BM) [8], [9]. One of the advantages of the BM is allowing independent models (disaggregate bundles) for an objective function given by a sum of separate terms [10], [11], as is the case in Unit Commitment problems. When some of these models (components) are simple to solve (continuous and linear optimization problems) they can be treated in a special way by basically copying the corresponding constraints in the formulation of the master problem, instead of iteratively approximating them by inner linearization. This has so far been applied to different problems [12], but we will show that the approach is very useful for Stochastic Unit Commitment (SUC) problems. In SUC, the goal is finding a production schedule that satisfies the unit's and system constraints considering the uncertainties, which in our case are related to inflows and demand. This optimization model is a step of the planning studies, where the system operator needs to determine the power plants operation for a day-ahead in a hydrothermal power system. The resulting problem is a large-scale, non-convex MINLP, which is extremely challenging to solve.

In the literature, there are basically two kinds of decomposition strategies based on LR for the SUC: Scenario Decomposition (SD), and Unit (or Space) Decomposition (UD). The SD references [13], [14] separate the stochastic problem in many sets of deterministic subproblems, using methods such as Progressive Hedging [15] and Branch and Bound [16] combined with LR. The UD references [17], [18] rather decompose the problem by power plant, using stochastic Lagrange multipliers related to the expected value of each scenario. Different techniques exist to represent the uncertainties, such as chance constraints [19]. The work [20] compares different approaches to represent the uncertainties in large scale problems. The extensive review [19] presents and describes the main strategies to model and to solve the SUC.

The aim of this paper is to compare the two main decomposition strategies, SD and UD, using the same algorithm to solve the SUC. Therefore, we avoid biased results, since we are applying and setting the same solver for both. Besides, we use several different cases, considering different configuration of power plants, in terms of capacity and number of units. In addition, we assess the impact of the "easy component" technique [12] to improve the convergence for some kinds of decomposition that have continuous and linear problems. The results are assessed by the lower bound, quality of the solutions provided by Lagrangian heuristics, and running time.

This paper is organized as follows: in sections II and III we describe the mathematical representation of the SUC and briefly reference and comment the data for all the test cases. In section IV, we present several variants of the decomposition strategies for the SUC, i.e., UD and SD. The computational comparison between the decomposition is presented in section V. Finally, in section VI, we state the conclusion and some recommendations regarding the decomposition approaches.

\section{UNIT COMMITMENT DESCRIPTION DATA}

A very common problem in power systems operation is to determine in advance which generating units will operate and their level of generation for the day ahead. This depends on the electrical energy market regulation, i.e., if the generation of the plants is defined by an Independent System Operator (ISO) centrally or by offer bids. This paper deals with the first case, such as the Brazilian one, in which the ISO executes a series of planning studies. The Unit Commitment (UC) problem is the last part of these studies, closer to the real-time operation, so a detailed mathematical model is required. In this section, we present the mathematical representation of each component of the power system, while in the following section we discuss the complete formulation for a general hydrothermal SUC problem.

For our study we use six hydrothermal test systems defined in Table I, considering different power capacity and number of units. The systems A to E have the same transmission system, with 46 buses and 95 lines, used for Sections VI-A to VI-C. System DD is larger, it has an 82-bus and 143-lines transmission system and is discussed in Section VI-D. The hydro plants are based on real information of the Brazilian system, where the data have been extracted from a data base of the HydroByte software [21]. The thermal plants data is instead taken from the UC instances of [22]. Finally, the transmission system and demand data for each bus were adapted from an equivalent system from the south of the Brazilian electric power system. All the data is available at http://www.di.unipi.it/optimize/Data/UC.html. We now discuss the mathematical representation and a de- 
scription of the data for each test system.

$$
\text { TABLE I }
$$

TEST SYSTEMS FOR HYDROTHERMAL SUC PROBLEMS

\begin{tabular}{ccccccc}
\hline \hline & $\begin{array}{c}\text { Number of } \\
\text { plants }\end{array}$ & \multicolumn{2}{c}{$\begin{array}{c}\text { Generation } \\
\text { capacity (\%) }\end{array}$} & $\begin{array}{c}\text { Generation } \\
\text { capacity (MW) }\end{array}$ & $\begin{array}{c}\text { Storage } \\
\text { capacity (hm }\end{array}$ \\
\hline \multicolumn{6}{c}{ ) } \\
A & 7 & 14 & 25.0 & 75.0 & $21,297.5$ & $5,635.1$ \\
B & 7 & 14 & 75.0 & 25.0 & $9,224.0$ & $9,309.0$ \\
C & 10 & 10 & 50.0 & 50.0 & $16,132.2$ & $10,737.5$ \\
D & 14 & 7 & 76.3 & 23.7 & $16,046.5$ & $14,944.1$ \\
E & 14 & 7 & 25.2 & 74.8 & $9,671.0$ & $5,507.2$ \\
DD & 28 & 15 & 74.8 & 25.2 & $29,922.6$ & $44,043.99$ \\
\hline \hline
\end{tabular}

$\mathrm{H}$ stand for hydro and $\mathrm{T}$ for thermoelectric power plants.

\section{A. Thermal Power Plants}

Thermal production cost depends on the fuel cost and varies accordingly to the power generation, following a convex quadratic relationship. This nonlinear relation is approximated using the perspective cuts approach [23], resulting in linear constraints and a variable to represent the operational cost of each thermal plant. The thermal operation has some technical conditions that must be satisfied, such as generation limits, minimum up and down times and ramp generation limits. All these constraints are represented by means of what are currently considered the best mixed-integer linear formulation for thermal units [24], [25], as shown in the Appendix. We succinctly denote the corresponding set of constraints as

$$
C^{\mathrm{T}}(p t, u, u p, u d, F) \text {. }
$$

\section{B. Hydro Power Plants}

Hydro plants produce energy using the potential energy of the water in the reservoir. The energy transformation process depends on the net head, the turbine and generation efficiencies and the units' turbined outflow. This complex relationship results in a non-convex function [26] that in this paper is simplified to mixed-integer linear constraints. This simplification includes the representation of a group of identical units by a single equivalent unit and a piecewise linearization of the production function for each equivalent unit. The following figures illustrate the simplified representation; better ones could be used, as in [27]-[29]. Figure 1 represents the power generation for a hydro plant with four identical units, while Figure 2 presents the final piecewise-linear nonconvex model that we have used.

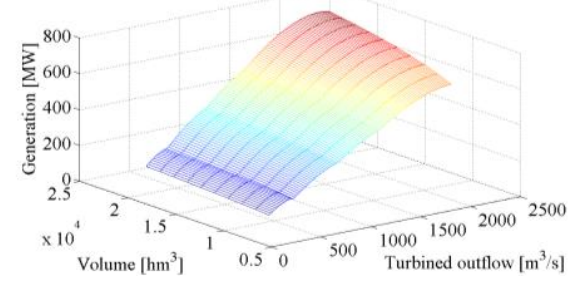

Fig. 1. Equivalent nonconvex production function.

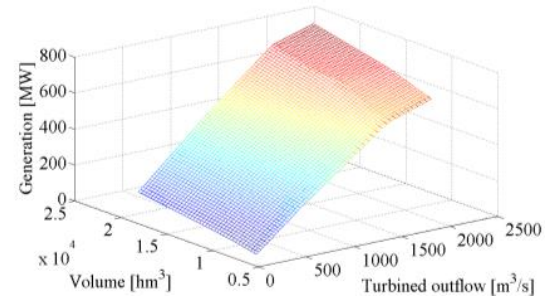

Fig. 2. Linear piecewise model of the equivalent unit.

The hydraulic connection between reservoirs (water flow balance equation) and the operational limits are taken into account as standard mixed-integer linear equations [26], [30]. The set of hydro constraints is denoted by

$$
C^{\mathrm{H}}(p h, v, d, s, p h g, q, z) \text {. }
$$

The operation of the reservoirs is coordinated with medium-term scheduling problem by means of target volumes at the end of the scheduling horizon, represented by linear constraints that are also included in (2).

\section{Transmission network}

The electrical network constraints are represented by DC power flow equations, where are included load and spinning reserve requirements. In Brazil, only hydro plants provide spinning reserve. The transmission network constraints are denoted by

$$
C^{\mathrm{D}}(p t, p h)
$$

\section{Two-Stage Unit Commitment Model}

The hydrothermal SUC aims at finding the optimal generation schedule while meeting operational and system wide constraints at a minimum expected cost. The latter takes into account a level of uncertainty due to the high dependence of the hydro production and the demand on the weather conditions. Indeed, although Brazil have large reservoirs, approximately 55\% [31] of the hydro plants are run-of-river ones, which strongly depend on the inflow to generate, so uncertainties on its values directly affect the operation of the power system. The uncertain data are the system load requirements (set $\mathrm{C}^{\mathrm{D}}$ ) and the water inflows (set $\mathrm{C}^{\mathrm{H}}$ ) represented by scenario trees. Figure 3 illustrates the uncertainty: two realizations of inflow and load profile result in four scenarios in the second stage. Each stage refers to 24 hours, representing the operation of one day. As a consequence, the corresponding scenario tree has a total of 120 nodes.

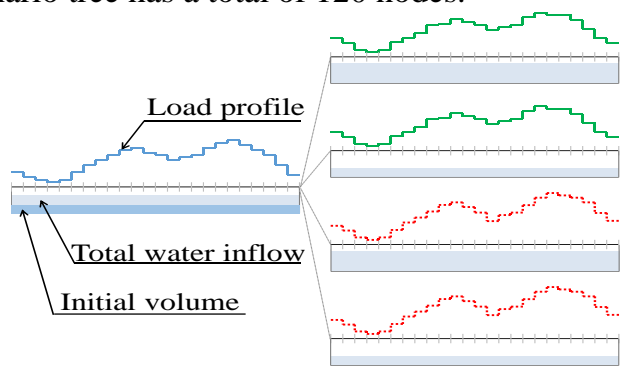

Fig. 3. Illustration of the uncertain data.

The model for the hydrothermal SUC problem is given by: 


$$
\begin{aligned}
& \min =\sum_{n=1}^{\mathrm{N}} p_{n} \cdot f_{n}\left(u p_{i n}, F_{i n}\right) \\
& \text { s.t.: } \quad C_{n}^{\mathrm{H}}\left(p h_{r n}, v_{r n}, d_{r n}, s_{r n}, p h g_{j m}, q_{j m}, z_{j m}\right), \quad \forall n \\
& C_{i n}^{\mathrm{T}}\left(p t_{i n}, u_{i n}, u p_{i n}, u d_{i n}, F_{i n}\right), \quad \forall n, i \\
& C_{n}^{\mathrm{D}}\left(p t_{i n}, p h_{r n}\right) \text {. } \\
& \forall n
\end{aligned}
$$

In the compact formulation (4)-(5), each vector variable is related to a specific node of the scenario tree. For instance, variable $p h_{r n}$ represents the power of hydro plant $r$ and node $n$. The complete formulation of the optimization model and the composition of each set of constraints are presented in the Appendix.

\section{DeCOMPOSITION STRATEgIES}

Applying LR to problem (4)-(5) can be done in different ways. The most common approaches are the unit decomposition (UD) and the scenario decomposition (SD). In the former, the whole problem is splitted by its physical characteristics, typically a subproblem for each power plant. On the other hand, the SD separates the stochastic problem in many deterministic UC subproblems, each one related with a specific scenario. The different strategies are illustrated in the Figure 4, which are described in the next sections.
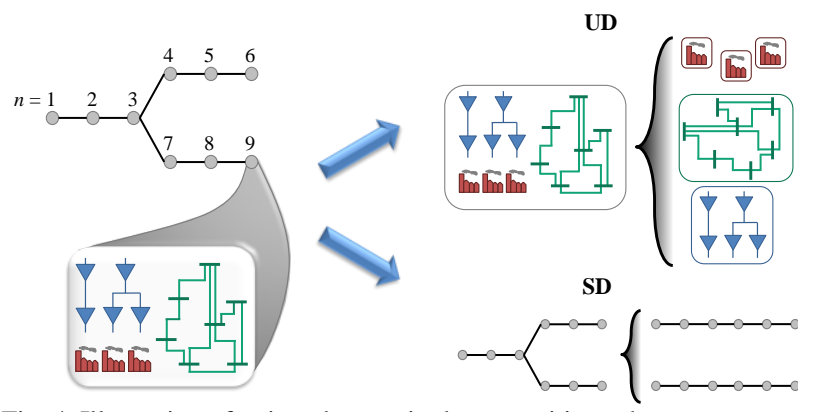

Fig. 4. Illustration of unit and scenario decomposition schemes.

\section{A. Unit Decomposition}

Considering the coupling constraints of problem (4)-(5), the most logical is to separate the problem by it characteristics: a set of subproblems for thermal plants, another set for all the hydro plants, and yet another set for the transmission network. Further, each set of subproblems can be divided even more. Given the predominance of hydro generation in Brazil, we propose three schemes for the UD. These schemes are illustrated in Figure 5 for a problem with threetime periods in each stage, two scenarios, five hydro plants (located in two cascades), three thermal plants and an electrical network with seven buses and ten lines. The continuous line represents the time coupling between each node (in this paper, one-hour period), and dotted line indicates that there is not time coupling.

The first scheme (UD1) splits problem (4)-(5) in:

a) many Linear Programing (LP) subproblems, representing the electrical network constraints;

b) many Mixed-Integer Linear Programming (MILP) subproblems, each one representing a thermal plant;

c) a MILP subproblem, coupled in time and space, rep- resenting the operation of all the hydro plants.
UD1

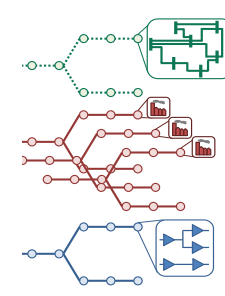

UD2

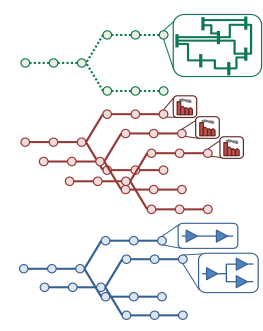

UD3

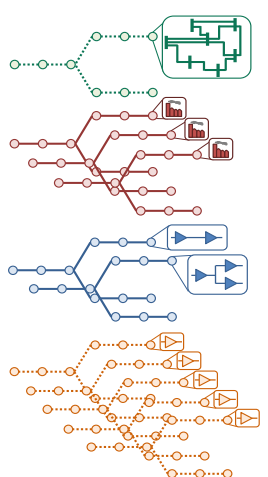

Fig. 5. Illustration of different kinds of unit decomposition.

Figure 6 shows coupling structure of UD1.

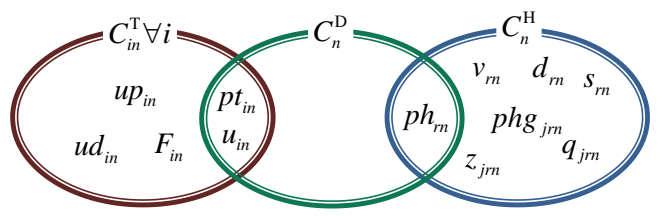

Fig. 6. Illustration of the relationship between variables for UD1.

To decompose the problem, we apply the variable splitting technique [32], resulting in the following dual problem:

$\Phi^{\mathrm{UDI}}(\lambda p t, \lambda p h)=\min f_{n}\left(u p_{i n}, F_{i n}\right)+$

$$
\begin{aligned}
& \sum_{n=1}^{\mathrm{N}}\left(\sum_{i=1}^{\mathrm{1}} \lambda p t_{i n} \cdot\left(p t a_{i n}-p t_{i n}-u_{i n} \cdot p t_{i}^{\mathrm{min}}\right)+\right. \\
& \left.\sum_{r=1}^{\mathrm{R}} \lambda p h_{r n} \cdot\left(p h a_{m}-p h_{r n}\right)\right) \\
& \text { s.t.: } \quad C_{n}^{\mathrm{H}}\left(p h_{r n}, v_{r n}, d_{r n}, s_{r n}, p h g_{j r n}, q_{j r n}, z_{j r n}\right), \quad \forall n \\
& C_{i n}^{\mathrm{T}}\left(p t_{i n}, u_{i n}, u p_{i n}, u d_{i n}, F_{i n}\right), \quad \forall n, i \\
& C_{n}^{\mathrm{D}}\left(p t a_{i n}, p h a_{m}\right), \quad \forall n
\end{aligned}
$$

The dual function (6) can be evaluated by means of $(1+\mathrm{I}+\mathrm{N})$ independent subproblems.

$\Phi^{\mathrm{UD} 1}=\Phi^{\mathrm{H}}+\sum_{i=1}^{\mathrm{I}} \Phi_{i}^{\mathrm{T}}+\sum_{n=1}^{\mathrm{N}} \Phi_{n}^{\mathrm{D}}$

The second scheme (UD2), which derives from UD1, is obtained applying the same decomposition and relaxing the spinning reserve constraints. The set of subproblem a) and b) are the same, but the subproblem c) changes as follows:

d) a set of MILP subproblems, representing the operation of the hydro plants for each cascade.

In this case, besides the strategy used in UD1, the spinning reserve constraint is relaxed applying the classical LR technique. Despite this constraint formally belongs to the set $C^{\mathrm{D}}$, it just couples the operation of hydro plants.

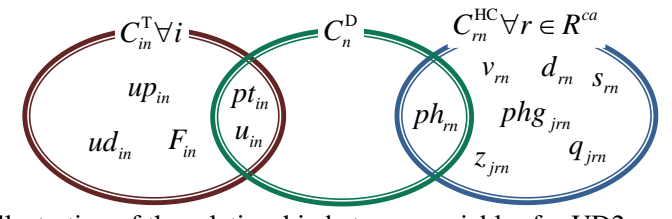

Fig. 7. Illustration of the relationship between variables for UD2.

The new set for hydro constraints $\mathrm{C}^{\mathrm{HC}}$ in Figure 7 is de- 
fined for each cascade, as shown in (9)-(10).

$$
\begin{aligned}
& \Phi^{\mathrm{UD} 2}(\lambda p t, \lambda p h, \lambda \text { res })=\min f_{n}\left(u p_{\text {in }}, F_{\text {in }}\right)+ \\
& \sum_{n=1}^{\mathrm{N}}\left(\sum_{i=1}^{\mathrm{I}} \lambda p t_{i n} \cdot\left(p t a_{i n}-p t_{i n}-u_{i n} \cdot p t_{i}^{\mathrm{min}}\right)+\right. \\
& \sum_{r=1}^{\mathrm{R}} \lambda p h_{r n} \cdot\left(p h a_{r m}-p h_{r n}\right)+ \\
& \left.\lambda \operatorname{res}_{n} \cdot\left(\sum_{r=1}^{\mathrm{R}}\left(\sum_{j=1}^{\mathrm{J}_{r}} p h g_{j r}^{\max } \cdot z_{j r m}-p h_{r m}\right)-\operatorname{Res}_{n}\right)\right) \\
& \text { s.t.: } C_{r m}^{\mathrm{HC}}\left(p h_{r n}, v_{r n}, d_{r n}, s_{r m}, p h g_{j m}, q_{j m}, z_{j m}\right), \forall n, r \in R^{\mathrm{CA}} \\
& C_{i n}^{\mathrm{T}}\left(p t_{i n}, u_{i n}, u p_{i n}, u d_{i n}, F_{i n}\right), \quad \forall n, i \\
& C_{n}^{\mathrm{D}}\left(p t a_{i n}, p h a_{m}\right), \quad \forall n
\end{aligned}
$$

The dual function in (9) can be evaluated by $(\mathrm{CA}+\mathrm{I}+\mathrm{N})$ independent subproblems, as follows:

$$
\Phi^{\mathrm{UD} 2}=\sum_{c a=1}^{\mathrm{CA}} \Phi_{c a}^{\mathrm{HC}}+\sum_{i=1}^{\mathrm{I}} \Phi_{i}^{\mathrm{T}}+\sum_{n=1}^{\mathrm{N}} \Phi_{n}^{\mathrm{D}}-\sum_{n=1}^{\mathrm{N}} \lambda \operatorname{res}_{n} \cdot \operatorname{Res}_{n}
$$

The third scheme (UD3 - Figure 8), which derives from UD2, separates even more the hydro set of subproblems. In UD3, the set of subproblems a) and b) are the same, but the set of subproblems d) in UD2 changes to:

e) a set of LP subproblems, representing the constraints between the reservoirs for each cascade;

f) a set of MILP subproblems, each one representing the operation of a hydro plant.

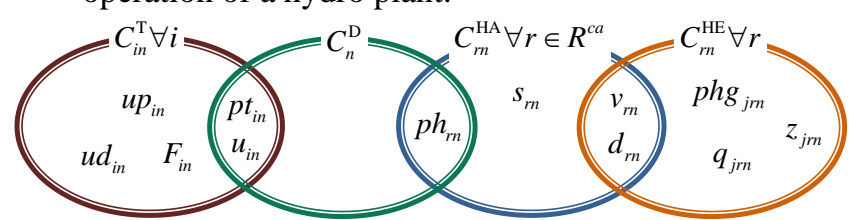

Fig. 8. Illustration of the relationship between variables for UD3.

To achieve UD3 we use the same relaxation strategy used in UD2, but splitting two additional variables: $v$ and $d$. The new sets $\mathrm{C}^{\mathrm{HA}}$ and $\mathrm{C}^{\mathrm{HE}}$, shown in Figure 8, derive from $\mathrm{C}^{\mathrm{H}}$ without the spinning reserve constraint. The first represents hydraulic constraints of the reservoirs, defined for each cascade, and $\mathrm{C}^{\mathrm{HE}}$ represent the constraints of the operation for each hydro plant. Mathematically, UD3 is given by:

$$
\begin{aligned}
& \Phi^{\mathrm{UD} 3}(\lambda p t, \lambda p h, \lambda \text { res }, \lambda v, \lambda d)=\min f_{n}\left(u p_{\text {in }}, F_{\text {in }}\right)+ \\
& \sum_{n=1}^{\mathrm{N}}\left(\sum_{i=1}^{\mathrm{I}} \lambda p t_{i n} \cdot\left(p t a_{i n}-p t_{i n}-u_{i n} \cdot p t_{i}^{\mathrm{min}}\right)+\right. \\
& \sum_{r=1}^{R}\left(\lambda p h_{r n} \cdot\left(p h a_{r n}-p h_{r n}\right)+\lambda v_{r n} \cdot\left(v a_{r n}-v_{r n}\right)+\right. \\
& \lambda d_{r n} \cdot\left(d a_{r n}-d_{r n}\right)+ \\
& \left.\operatorname{res}_{n} \cdot\left(\sum_{r=1}^{\mathrm{R}}\left(\sum_{j=1}^{\mathrm{J}_{r}} p h g_{j r}^{\max } \cdot z_{j r n}-p h_{r n}\right)-\operatorname{Res}_{n}\right)\right) \\
& \text { s.t.: } C_{r n}^{\mathrm{HA}}\left(v a_{r n}, d a_{r n}, s_{r n}\right), \quad \forall n, r \in R^{\mathrm{CA}} \\
& C_{r n}^{\mathrm{HE}}\left(p h_{r n}, v_{r n}, d_{r n}, s_{r n}, p h g_{j r n}, q_{j r n}, z_{j r n}\right) \forall n, r \\
& C_{i n}^{\mathrm{T}}\left(p t_{i n}, u_{i n}, u p_{i n}, u d_{i n}, F_{i n}\right), \quad \forall n, i \\
& C_{n}^{\mathrm{D}}\left(p t a_{i n}, p h a_{r n}\right), \quad \forall n
\end{aligned}
$$

The dual function (11) can be evaluated $(\mathrm{CA}+\mathrm{N} \cdot \mathrm{R}+\mathrm{I}+\mathrm{N})$ independent subproblems.

$$
\begin{aligned}
& \Phi^{\mathrm{UD} 3}= \\
& \sum_{c a=1}^{\mathrm{CA}} \Phi_{c a}^{\mathrm{HA}}+\sum_{n=1}^{\mathrm{N}} \sum_{r=1}^{\mathrm{R}} \Phi_{r m}^{\mathrm{HE}}+\sum_{i=1}^{\mathrm{I}} \Phi_{i}^{\mathrm{T}}+\sum_{n=1}^{\mathrm{N}} \Phi_{n}^{\mathrm{D}}-\sum_{n=1}^{\mathrm{N}} \lambda r e s_{n} \operatorname{Res}_{n}
\end{aligned}
$$

\section{B. Scenario Decomposition}

This strategy separates SUC in single-scenario deterministic subproblems, applying the variable splitting technique in the linking variables of the first stage; in other words, the non-anticipativity constraints are relaxed. In this case, the constraints are rearranged and the sets are separated by scenario. For instance, all the constraints belonging to nodes of the scenario one, $N(1)$, make up $\mathrm{C}_{1}^{\mathrm{C}}$, which is derived from $\mathrm{C}^{\mathrm{T}}, \mathrm{C}^{\mathrm{H}}$ and $\mathrm{C}^{\mathrm{D}}$ regarding the nodes of scenario one. Figure 9 illustrates the sets of constraints for the problem of Figure 4, representing the variables that couples the subproblems. The set $N_{x}$ represents all the nodes of period $x$.

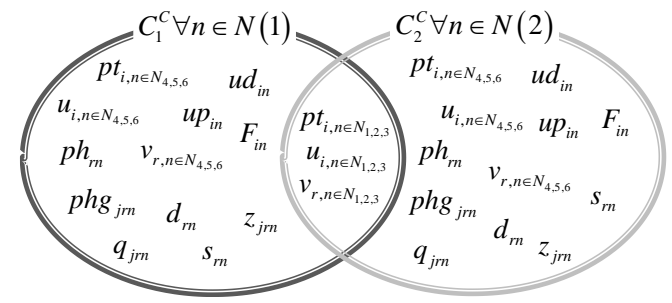

Fig. 9. Illustration of the relationship between variables for SD.

Relaxing the non-anticipatively constraints results in the following dual problem:

$$
\begin{aligned}
& \Phi^{\mathrm{SD}}(\gamma p t, \gamma u, \gamma v)=\min f_{n}\left(u p_{i n}, F_{i n}\right)+ \\
& \sum_{\omega=1}^{\Omega}\left(\sum _ { n \in N ( \omega ) } \left(\sum _ { i = 1 } ^ { I } \left(\gamma p t_{i n} \cdot\left(p t_{i n}-\sum_{\sigma=1}^{\Omega} p_{\sigma} \cdot p t_{i, m \in N_{t} \cap N(\sigma)}\right)+\right.\right.\right. \\
& \left.\gamma u_{i n} \cdot\left(u_{i n}-\sum_{\sigma=1}^{\Omega} p_{\sigma} \cdot u_{i, m \in N_{t} \cap N(\sigma)}\right)\right)+ \\
& \left.\left.\sum_{r=1}^{R} \gamma v_{r n} \cdot\left(v_{r n}-\sum_{\sigma=1}^{\Omega} p_{\sigma} \cdot v_{r, m \in N_{t} \cap N(\sigma)}\right)\right)\right) \\
& \text { s.t.: } C_{\omega}^{\mathrm{C}}\left(\begin{array}{l}
\left.p t_{i n}, u_{i n}, u p_{i n}, u d_{i n}, F_{r n}, p h_{r n}, v_{r n}, d_{r n}, s_{r n},\right), \\
p h g_{j r n}, q_{j r n}, z_{j r n}
\end{array}\right) \\
& \forall i, r, b, n \in N(\omega), \omega=1, \ldots, \Omega
\end{aligned}
$$

The dual function (13) can be evaluated by several onescenario subproblems $(\Omega)$, as follows:

$$
\Phi^{\mathrm{SD}}=\sum_{\omega=1}^{\Omega} \Phi_{\omega}^{\mathrm{C}}
$$

\section{Algorithm}

The Lagrangian duals corresponding to all of the strategies described in the previous section are solved by means of a Bundle-type method [33]. In particular, it is a "generalized" Bundle method [34], in that the Master Problem (MP) does not necessarily need to be a Quadratic Program. For this work, we used the linear (box) stabilizing term, which is known to significantly reduce master problem time w.r.t. the usual quadratic term [12]. Solving the MP provides an estimate of the optimal Lagrangian multipliers that are fed to the 
subproblems, which in turn provide dual function values and subgradients that are used to update the MP, iterating the process until convergence is attained. The problem solved in this work has a dual function with a disaggregated structure, i.e., the dual function is a sum of many components, as can be seen in (8), (10), (12) and (14), and in similar works [10]. The Bundle algorithm is implemented in a $\mathrm{C}++$ code developed by the third author, which is available upon request. Both the MP and the subproblems are solved by means of a general optimization solver. Table II presents, for the different strategies, the size of the dual problem and associated subproblems.

TABLE II

CHARACTERISTIC OF THE DECOMPOSITION STRATEGIES

\begin{tabular}{cccc}
\hline \hline Decomposition & Number of dual variables & \multicolumn{2}{c}{ Number of subproblems } \\
\hline & & LP & MILP \\
UD1 & $\mathrm{N} \cdot(\mathrm{I}+\mathrm{R})$ & $\mathrm{N}$ & $1+\mathrm{I}$ \\
UD2 & $\mathrm{N} \cdot(\mathrm{I}+\mathrm{R}+1)$ & $\mathrm{N}$ & $\mathrm{CA}+\mathrm{I}$ \\
UD3 & $\mathrm{N} \cdot(\mathrm{I}+1+3 \cdot \mathrm{R})$ & $\mathrm{N}+\mathrm{CA}$ & $\mathrm{N} \cdot \mathrm{R}+\mathrm{I}$ \\
SD & $\mathrm{T}_{1} \cdot \Omega \cdot(2 \cdot \mathrm{I}+\mathrm{R})$ & 0 & $\Omega$ \\
\hline \hline
\end{tabular}

$\mathrm{T}_{1}$ is the number of nodes in stage 1.

The disaggregated model allows to use the "easy component" technique [12], whereby some of the components (subproblems), the "easy" ones, are included into the MP. Without this technique, the subproblems are modeled in the MP as piecewise-linear approximations, which are improved throughout the iterations. On the other hand, using the technique continuous and linear subproblems are actually modeled in the MP, and consequently solved exactly. This increases its size, but provides an exact description of the easy components, instead of an approximated iteratively refined by means of cuts. All the LP subproblems are candidate to be treated as easy components.

\section{RESULTS}

All the results have been obtained on an Intel Xeon CPU X5690 (3.47 GHz) computer with 32.0 GigaBytes of RAM. The LP and MILP subproblems are solved using the 6.0.5 Gurobi optimization solver. In the tests, we have various hydrothermal systems, different initial conditions and distinct scenarios trees to produce a wide range of results. The results are compared mainly using the performance profile technique [35]. It allows defining a distribution function for some metric (number of iteration, processing time, quality of the objective function, etc.) comparing algorithms by means of this metric. The performance profiles are cumulative distribution functions for a metric, which depend on the multiplying factor, i.e., is the relation between the current method and the best one.

$\Phi_{X}(f m)=\frac{\text { number of cases which } \eta_{X}(p c) \leq f m \cdot \eta^{*}(p c)}{\text { total of evaluated cases }}$

The results of the tests are presented in the next sections using these performance profiles and the usual statistical metrics (average values and standard deviation).

\footnotetext{
${ }^{2}$ We tested with the latest version of Gurobi, 7.5, and the results are completely analogous.
}

\section{A. Easy components}

The goal of this section is to explore the effect of applying the easy component technique. We consider 30 cases: 15 deterministic cases and 15 stochastic ones. The 15 are different regarding the kind of system (five hydrothermal systems, A to E) and the initial conditions (three initial volume conditions). For this test, we compare the two kinds of decomposition where the use of easy components is possible and result in more impact: UD2 and UD3. The subproblems $\Phi^{\mathrm{D}}$ and $\Phi^{\mathrm{HA}}$ are modeled as easy components in the current work, they are composed by constraints (38) to (40) and (18) to (20), respectively. Figure 10 report the performance profile for the number of iterations, where "ccs" is the variant with easy component, while "scs" (dashed) is the one without it. Figure 10 shows that the use of easy component does not have a major impact in UD2, although on average it results $18 \%$ less iterations. The impact for UD3 is much more significant: in about $85 \%$ of the cases there are less iterations, and more importantly, the average reduction of iterations in UD3 is around $600 \%$. In terms of computational times, using easy components on average reduces them by $22 \%$ and $2,544 \%$, for UD2 and UD3, respectively. Since this technique is clearly beneficial, in all the tests presented in the following sections it is applied.

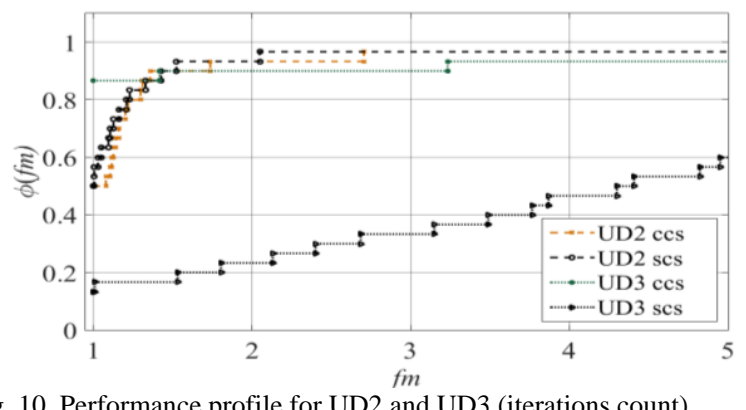

Fig. 10. Performance profile for UD2 and UD3 (iterations count).

\section{B. Deterministic instances}

In this section we present a comparison between the decomposition strategies using deterministic data for five test systems (A to E in Table I) with three initial conditions, resulting in 15 cases. We present the average values (and standard deviation, in brackets) in Table III. Since LR is a relaxation, which does not solve MILP problems to primal optimality, all the unit decomposition schemes are compared by means of the quality of the obtained bound, measured by the duality gap $\left(g_{a p}\right)$ and gap associated with the continuous relaxation solution $\left(\mathrm{gap}_{2}\right)$, given by

$g_{a p}=\frac{U B-L B}{L B}, \operatorname{gap}_{2}=\frac{L B-C R}{C R}$,

where the upper bound $U B$ is best known solution for the problem found by running a MILP solver for 10 hours, the lower bound $L B$ is the optimum of the corresponding Lagrangian Dual, and $C R$ is the lower bound provided by the continuous relaxation. We also present the total processing time, the oracle time and the number of iterations for all the schemes. 
TABLE III

RESULTS FOR DETERMINISTIC CASES

\begin{tabular}{lccccc}
\hline \hline Strategy & gap $_{1}[\%]$ & gap $_{2}[\%]$ & Time* $^{*}$ & Oracle time [\%] & Iterations* \\
\hline UD1 & $1(2)$ & $126(314)$ & $9(16)$ & $88(10)$ & $4(5)$ \\
UD2 & $2(4)$ & $137(290)$ & $10(9)$ & $86(16)$ & $2(2)$ \\
UD3 & $5(5)$ & $122(259)$ & $1(0.9)$ & $39(12)$ & $1(0.9)$ \\
\hline \hline
\end{tabular}

The value within brackets represents the standard deviation.

*The time and the number of iterations are presented with regard to the smaller values, which are the ones obtained by UD3. Time $=1$ corresponds to $3 \mathrm{~min}$. and Iteration $=1$ corresponds to 84 .

The table shows a clear trade-off between running time and quality of the obtained bound. Strategy UD3 is an order of magnitude faster than the others; while it also provide bounds of lesser quality, the difference in the gaps is comparatively smaller than that in running rimes. The main reason for this large advantage is the difficulty of the subproblem that represents the hydro constraints. In UD1 and UD2 this subproblem is rather complex and can consume almost all the time.

- For UD1, the Hydro and Thermal subproblems take on average $77 \%$ and $11 \%$ of the total time, respectively.

- For UD2, the averages are $77 \%$ and $9 \%$ regarding the Hydro subproblem and Thermal subproblem, respectively.

- For UD3, $12 \%$ is regarding the Hydro subproblem $\left(\Phi^{\mathrm{HE}}\right)$ and $27 \%$ regarding the Thermal subproblem.

The other subproblems have no relative time in the Oracle since they are solved as easy components, in the MP.

Indeed, Table III shows that for these strategies the fraction of time spent in solving the subproblems is much larger than for the UD3. Which strategy would be preferable depends on the way in which the LR is used to solve the original problem. If the bound were to be used within an implicit enumeration algorithm, then the better quality of UD1 and UD2 might be worth the extra running time. However, exact algorithms are typically too costly for this application, and the standard use of LR is rather to drive heuristic ones. For these, arguably the quality of the bound is less important than the overall running time, and therefore UD3 is likely to be preferable.

\section{Stochastic instances}

In this section, we compare all the strategies for stochastic data. We consider two sizes of scenario tree, with four and nine scenarios, for five test systems (A to E) with three initial conditions, resulting in 30 cases. Figures 11 and 12 show the performance profiles for the number of iterations and the processing times, respectively.

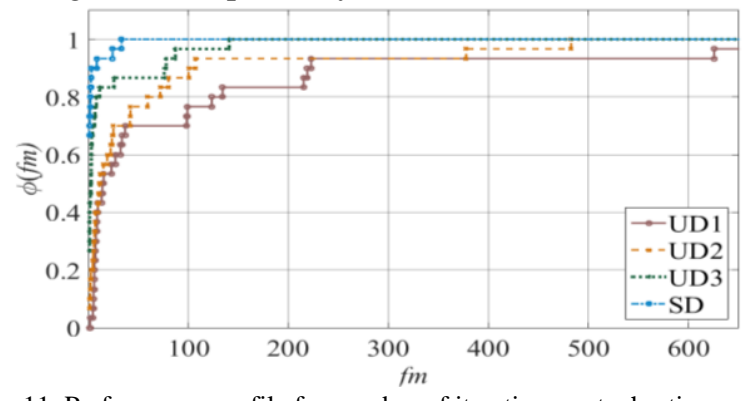

Fig. 11. Performance profile for number of iterations - stochastic cases.
Figure 11 shows that the SD converges in less iteration than the other methods in about $90 \%$ of the cases. However, its running time is one of the worst, as shown in Figure 12. This is due to the fact that it has to solve rather hard subproblems, which is where most of the time is spent. UD3 is still the best choice in terms of running times, with the tradeoff again being its lower bound, which worse than the others, as it can be seen in Figure 13. Conversely, SD results in the best lower bound in most cases. However, as we can see in Table IV (which presents the results by means of statistical metrics, similarly to Table III), the bounds found by the four strategies are close.

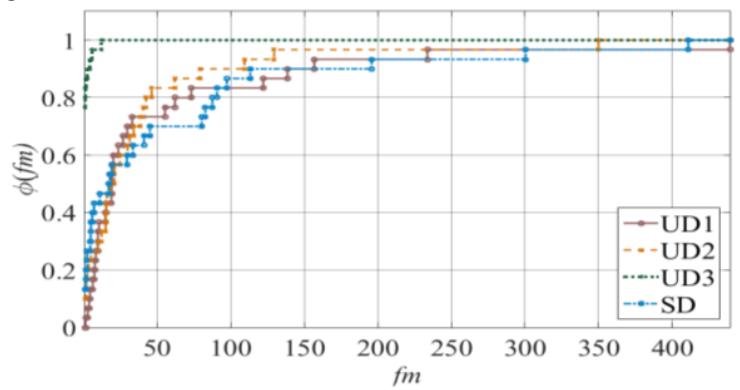

Fig. 12. Performance profile for processing time - stochastic cases.

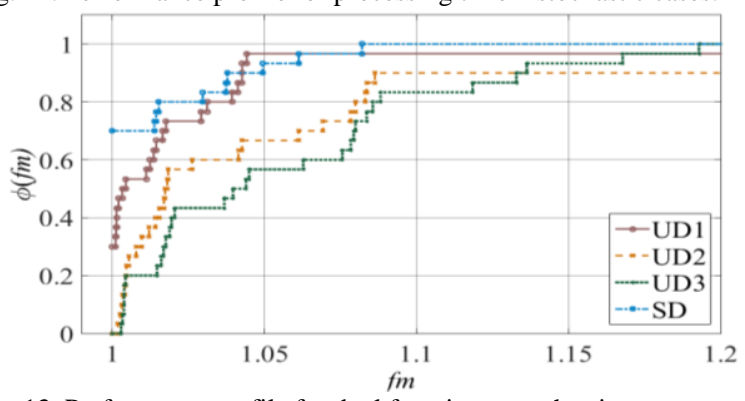

Fig. 13. Performance profile for dual function - stochastic cases.

TABLE IV

RESUlTS FOR STOCHASTIC CASES

\begin{tabular}{lrrrrr}
\hline \hline Strategy & Gap1 [\%] & Gap2 [\%] & Time* $^{*}$ Oracle time [\%] & Iterations* \\
\hline UD1 & $2(2)$ & $169(518)$ & $21(19)$ & $70(13)$ & $32(18)$ \\
UD2 & $10(21)$ & $142(287)$ & $11(9)$ & $80(21)$ & $12(8)$ \\
UD3 & $7(7)$ & $171(370)$ & $1(1.3)$ & $24(8)$ & $4(4)$ \\
SD & $2(2)$ & $198(425)$ & $10(7)$ & $100(1)$ & $1(0.6)$ \\
\hline UD1 & $4(3)$ & $20(47)$ & $13(10)$ & $49(21)$ & $8(7)$ \\
UD2 & $4(4)$ & $24(44)$ & $27(28)$ & $62(26)$ & $9(8)$ \\
UD3 & $7(7)$ & $123(378)$ & $1(1)$ & $16(6)$ & $1(0.9)$ \\
SD & $2(2)$ & $143(436)$ & $16(10)$ & $99(1)$ & $1(0.6)$ \\
\hline \hline
\end{tabular}

The value within brackets represents the standard deviation.

*The time and the number of iterations are presented with regard to the smaller values, i.e., UD3 for the time and SD for the iterations. Time $=1$ corresponds to 19 and $76 \mathrm{~min}$., for 4 and 9 scenarios, respectively. Iteration $=1$ corresponds to 28 and 80 , for 4 and 9 scenarios, respectively.

As the table IV shows, two strategies stand out. The SD presents the smaller values for gap $_{1}$, with a processing time that is close to that of UD1. On the other hand, the UD3 presents by far the smaller processing times, with a gap $_{1}$, slightly bigger than the others strategies, but still reasonable. We finish this section by presenting more detailed results regarding the distribution of power plants for each test system presented in Table I. Table V shows the values of gap $_{1}$, in \%, considering the deterministic and stochastic cases (but 
for the SD that is not applicable for deterministic instances). TABLE V

RESULTS FOR DIFFERENT SYSTEMS

\begin{tabular}{lrrrl}
\hline \hline System & \multicolumn{1}{c}{ UD1 } & \multicolumn{1}{c}{ UD2 } & \multicolumn{1}{c}{ UD3 } & SD \\
\hline A & $0.3(0.2)$ & $0.4(0.2)$ & $0.4(0.2)$ & $0.2(0.1)$ \\
B & $10.9(28.0)$ & $10.4(20.4)$ & $12.8(7.6)$ & $4.1(3.2)$ \\
C & $4.6(3.0)$ & $8.2(5.4)$ & $7.3(3.4)$ & $1.6(1.4)$ \\
D & $2.4(2.0)$ & $9.8(22.4)$ & $9.7(5.0)$ & $2.0(1.0)$ \\
E & $2.1(1.1)$ & \multicolumn{1}{c}{$2.4(0.8)$} & $2.1(0.8)$ & $0.8(0.4)$ \\
\hline \hline
\end{tabular}

The value within brackets represents the standard deviation.

As the Table V shows, systems with predominance of hydro plants (systems B, C and D) present larger values for the gap $_{1}$, and, in general, take more time to converge. Test system $\mathrm{B}$ results in the largest values of the gap $_{1}$ for all the strategies and large standard deviations due to the Hydro subproblem. In this kind of subproblem the quality of the solution depends on the availability of water for generation, the more water available more are the number of possible states to be visit by the solver. On the other hand, the system A, which has a larger participation of thermal plants presents the smallest values of gap $_{1}$.

\section{Results for System DD}

In this section, we compare the best performance strategies (UD3 and SD) for the System DD with stochastic data. We consider three initial conditions and twelve different scenario trees (with four, nine, sixteen and fifty-five scenarios and three different realizations for each one), resulting in 36 cases for the same system. Figures 14 and 15 show the performance profiles for the processing times and the dual function, respectively.

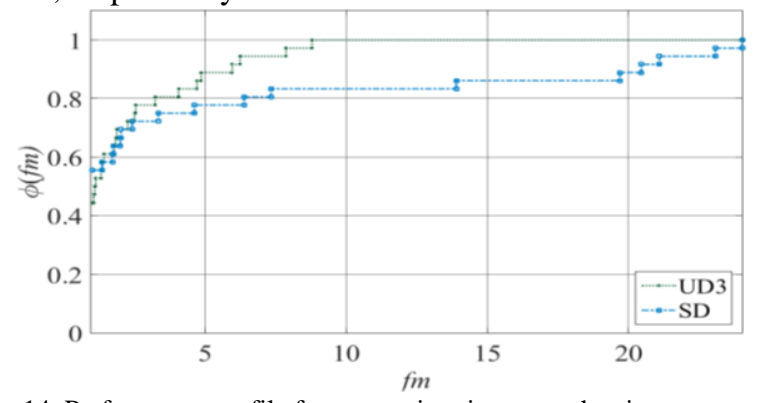

Fig. 14. Performance profile for processing time - stochastic cases.

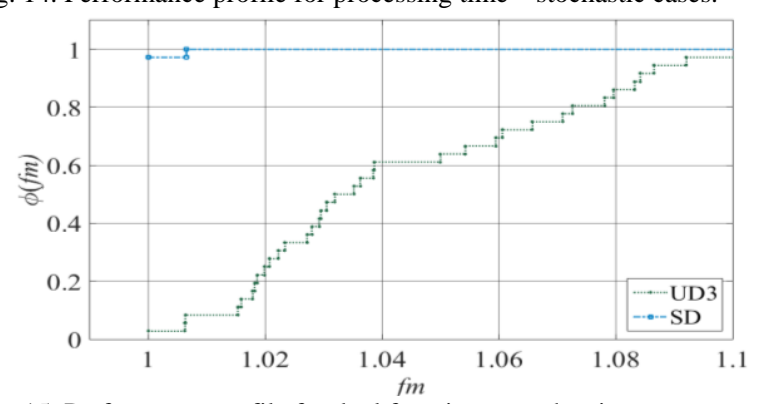

Fig. 15. Performance profile for dual function - stochastic cases.

We present the average values (and standard deviation) in Table VI.

The performance profile shows the UD3 presents slightly smaller times for stochastic cases. On the other hand, SD presents dual function values better, sometimes significantly so, than the one in UD3. Clearly, there are trade-offs be- tween the methods, which shows why choose the right decomposition is important. In Table VI, the value of gap ${ }_{1}$ for 16 and 25 scenarios is large due to the primal solution that was determined by a poor precision. On the other hand, the $\mathrm{Gap}_{2}$ can be used as metric for the quality of produced lower bound. Since the CR is the same for all decompositions, small values of $\mathrm{Gap}_{2}$ represent lesser improvements in the dual function, meanwhile large values means a better lower bound for the problem.

TABLE VI

RESULTS FOR STOCHASTIC CASES - SYSTEM DD

\begin{tabular}{|c|c|c|c|c|c|}
\hline Strategy & $\operatorname{Gap}_{1}[\%]$ & $\operatorname{Gap}_{2}[\%]$ & Time* & Oracle time [\%] & Iterations* \\
\hline \multicolumn{6}{|c|}{4 SCENARIOS } \\
\hline UD3 & $26(16)$ & $125(32)$ & $1.0(0.1)$ & $17.0(1.0)$ & $\overline{8.0(7.8)}$ \\
\hline SD & $24(16)$ & $117(25)$ & $3.8(4.6)$ & $99.9(0.0)$ & $1.0(1.0)$ \\
\hline \multicolumn{6}{|c|}{ 9 SCENARIOS } \\
\hline UD3 & $56(31)$ & $124(34)$ & $1.0(0.1)$ & $15.3(0.7)$ & $1.4(7.4)$ \\
\hline SD & $52(30)$ & $36(24)$ & $13.0(9.5)$ & $99.2(0.9)$ & $1.0(5.2)$ \\
\hline \multicolumn{6}{|c|}{16 SCENARIOS } \\
\hline UD3 & $2000(1009)$ & $133(32)$ & $1.4(0.2)$ & $13.2(1.3)$ & $24.6(42)$ \\
\hline SD & $1880(976)$ & $130(33)$ & $1.0(0.7)$ & $99.9(0.1)$ & $1.0(1.7)$ \\
\hline \multicolumn{6}{|c|}{25 SCENARIOS } \\
\hline UD3 & $2685(2501)$ & $47(35)$ & $2.3(0.3)$ & $10.6(1.2)$ & $21.6(21.0)$ \\
\hline SD & 2499 (2337) & $111(23)$ & $1.0(1.0)$ & $99.9(0.1)$ & $1.0(1.0)$ \\
\hline
\end{tabular}

\section{CONCLUSIONS}

Lagrangian Decomposition is a fundamental technique for solving very-large-scale, hard optimization problems like SUC and others [12]. It exploits the problem structure, splitting it in many subproblems. However, applications like SHUC have actually more than one forms of exploitable structure, such as unit and scenarios, each with possibly different variants. Although some theoretical guidelines exist [1], [19], choosing the best variant is never obvious, as complex trade-offs between bounds and iterations have to be taken into account. Although there are studies comparing different kinds of decomposition for the SUC problem [14], [18], to the best of our knowledge no one has compared the use of scenario and unit decomposition to the same UC problem, in particular with the three different variants of the Unit (Space) Decomposition and the use of "easy components". Our results show that Scenario Decomposition, although providing the best duality gap, is not competitive in terms of computational burden. On the other hand, UD, and in particular UD3 (using "easy components") has worse gaps, but only slightly so, while being much more efficient computationally. Furthermore, we have found that solution difficulty of dual problem depends on the amount of hydro in the system. This work provides solid foundations for a subsequent one, in which we will analyze the performance of the different decompositions schemes to obtain the primal solution of the problem. Besides LR, this requires other techniques, like inexact augmented Lagrangian [36] or Lagrangian Heuristics [5], [37], to construct the actual feasible solution required by the users. Solving the LR in this context has a specific set of 
trade-offs between bound quality, solution quality and solution time that require specific consideration. All this shows the importance to evaluate the strengths and weaknesses of the several different possible LR approaches.

\section{APPENDIX}

The complete formulation for the hydrothermal SUC problem is given by:

$$
\min =\sum_{n=1}^{\mathrm{N}} p_{n} \cdot \sum_{i=1}^{I}\left(F_{i n}+f_{i}^{3} \cdot u p_{\text {in }}\right)
$$

s.t:

$v_{r n}-v_{r n_{-1}}+0,0036 \cdot\left[d_{r n}-\sum_{r^{+} \in \Re_{r}^{+}} d_{r^{+} n_{-\tau}}\right]=0,0036 \cdot y_{r n}$,

$v_{r}^{\min } \leq v_{r n} \leq v_{r}^{\max }$,

$v_{r n} \geq v_{r}^{\text {target }}, n \in N_{L}$

$d_{r n}=s_{r n}+\sum_{j=1}^{J_{r}} q_{j r n}$,

$p h_{r n}=\sum_{j=1}^{J_{r}} p h g_{j r n}$

$s_{r n} \leq s_{r}^{\max }$,

$p h g_{j r n} \leq f p h g_{j r n}^{c h}\left(v_{r n}, q_{j r n}, d_{r n}, s_{r n}\right)+\left(1-z_{j r n}\right) \cdot p h g_{j r}^{\max }, c h \in C H_{j r}$

$p h g_{j r}^{\min } \cdot z_{j r n} \leq p h g_{j r n} \leq p h g_{j r}^{\max } \cdot z_{j r n}$,

$q_{j r}^{\min } \cdot z_{j r n} \leq q_{j r n} \leq q_{j r}^{\max } \cdot z_{j r n}$,

$z_{j r n} \in\{0,1\}$,

$\sum_{r=1}^{R}\left(\sum_{j=1}^{J_{r}} p h g_{j r}^{\max } \cdot z_{j r n}-p h_{r n}\right) \geq \operatorname{Res}_{n}$.

$F_{i n} \geq \alpha_{i}^{0, c f} \cdot\left(p t_{\text {in }}+u_{\text {in }} \cdot p t_{i}^{\mathrm{min}}\right)+\alpha_{i}^{1, c f} \cdot u_{i n}, c f \in C F_{i}$

$u_{i n}-u_{i n_{-1}}=u p_{i n}-u d_{i n}$,

$\sum_{n a=\max \left(1, n, t_{-t_{i} p}\right)}^{n} u p_{i, n a} \leq u_{i n}$

$\sum_{n a=\max \left(1, n-t_{-}^{d o w n}\right)}^{n} u d_{i, n a} \leq 1-u_{i n}$,

$p t_{i n}-p t_{i n_{-1}} \leq \Delta_{i}^{u p}$,

$-p t_{i n}+p t_{i n_{-1}} \leq \Delta_{i}^{d o w n}$

$0 \leq p t_{\text {in }} \leq p t_{i}^{\max }-p t_{i}^{\min }$,

$p t_{i n} \leq\left(p t_{i}^{\max }-p t_{i}^{\min }\right) \cdot\left(u_{i n}-u p_{i n}\right)$,

$$
\forall t_{i}^{u p}=0,
$$

$p t_{i n} \leq\left(p t_{i}^{\max }-p t_{i}^{\min }\right) \cdot\left(u_{i n}-u d_{i n_{+1}}\right)$,

$\forall t_{i}^{u p}=0$,

$p t_{i n} \leq\left(p t_{i}^{\max }-p t_{i}^{\min }\right) \cdot\left(u_{i n}-u p_{i n}-u d_{i n_{+1}}\right), \quad \forall t_{i}^{u p} \geq 1$,

$u_{i n}, u p_{i n}, u d_{i n} \in\{0,1\}$,

$\sum_{b=1}^{B} \sum_{r \in R_{b}} p h_{r n}+\sum_{b=1}^{B} \sum_{i \in I_{b}} p t_{i n}+u_{i n} \cdot p t_{i}^{\mathrm{min}}+\sum_{b=1}^{B} d e f_{b n}=\sum_{b=1}^{B} D_{b n}$, $-f l_{l}^{\max } \leq \sum_{b=1}^{B} \Gamma_{l b}\left(\sum_{r \in R_{b}} p h_{r n}+\sum_{i \in I_{b}} p t_{i n}+u_{i n} \cdot p t_{i}^{\min }+d e f_{b n}-D_{b n}\right)$,
$f l_{l}^{\max } \geq \sum_{b=1}^{B} \Gamma_{l b}\left(\sum_{r \in R_{b}} p h_{r n}+\sum_{i \in I_{b}} p t_{i n}+u_{i n} \cdot p t_{i}^{\min }+d e f_{b n}-D_{b n}\right)$,

In addition, the sets of constraints are structured as:

- Constraints (18) to (28) compose set $C_{n}^{\mathrm{H}}$.

- Constraints (29) to (37) compose set $C_{i n}^{\mathrm{T}}$.

- Constraints (38) to (40) compose set $C_{n}^{\mathrm{D}}$.

- Constraints (18) to (27) compose set $C_{r n}^{\mathrm{HC}}$.

- Constraints (18) to (20) compose set $C_{r n}^{\mathrm{HA}}$.

- Constraints (21) to (27) compose set $C_{r n}^{\mathrm{HE}}$.

- Constraints (18) to (40) compose set $C_{\omega}^{\mathrm{C}}$.

\section{REFERENCES}

[1] M. Guignard, "Lagrangean Relaxation," Top - Journal of the Spanish Society of Statistics and Operations Research, vol. 11, no. 2, pp. 151-228, 2003.

[2] C. Lemaréchal, "Lagrangian Relaxation," in Computational Combinatorial Optimization, M. Jünger and D. Naddef, Eds. Springer Berlin Heidelberg, 2001, pp. 112-156.

[3] M. L. Fisher, "An applications oriented guide to Lagrangean relaxation," Interfaces, vol. 15, pp. 10-21, 1985.

[4] M. L. Fisher, "The Lagrangian Relaxation Method for Solving Integer Programming Problems," Management Science, vol. 50, no. 12_supplement, pp. 1861-1871, Dec. 2004.

[5] L. Dubost, R. Gonzalez, and C. Lemaréchal, "A primalproximal heuristic applied to the French Unit-commitment problem," Mathematical Programming, vol. 104, no. 1, pp. 129-151, 2005.

[6] S. Cerisola, J. M. Latorre, and A. Ramos, "Stochastic dual dynamic programming applied to nonconvex hydrothermal models," European Journal of Operational Research, vol. 218, no. 3, pp. 687-697, May 2012.

[7] G. Steeger and S. Rebennack, "Dynamic convexification within nested Benders decomposition using Lagrangian relaxation: An application to the strategic bidding problem," European Journal of Operational Research, vol. 257, no. 2, pp. 669-686, Mar. 2017.

[8] P. Wolfe, "A method of conjugate subgradients for minimizing nondifferentiable functions," in Nondifferentiable Optimization, M. L. Balinski and P. Wolfe, Eds. Springer Berlin Heidelberg, 1975, pp. 145-173.

[9] C. Lemarechal, "An extension of davidon methods to non differentiable problems," in Nondifferentiable Optimization, M. L. Balinski and P. Wolfe, Eds. Springer Berlin Heidelberg, 1975, pp. 95-109.

[10] L. Bacaud, C. Lemaréchal, A. Renaud, and C. Sagastizábal, "Bundle methods in stochastic optimal power management: a disaggregated approach using preconditioners," Computational Optimization and Applications, vol. 20, pp. 227-244, 2001.

[11] Y. Zhang, N. Gatsis, and G. B. Giannakis, "Disaggregated bundle methods for distributed market clearing in power networks," in 2013 IEEE Global Conference on Signal and Information Processing, 2013, pp. 835-838.

[12] A. Frangioni and E. Gorgone, "Bundle methods for sumfunctions with 'easy' components: applications to multicommodity network design," Math. Program., vol. 145, no. 1-2, pp. 133-161, Jun. 2014. 
[13] S. Takriti, J. R. Birge, and E. Long, "A stochastic model for the unit commitment problem," Power Systems, IEEE Transactions on, vol. 11, no. 3, pp. 1497-1508, 1996.

[14] C. C. Carøe and R. Schultz, "A Two-Stage Stochastic Program for Unit Commitment under Uncertainty in a HydroThermal Power System.," Konrad-Zuse-Zentrum für Informationstechnik Berlin, Berlin, Germany, 1997.

[15] R. T. Rockafellar and R. J.-B. Wets, "Scenarios and Policy Aggregation in Optimization Under Uncertainty," Mathematics of OR, vol. 16, no. 1, pp. 119-147, Feb. 1991.

[16] A. H. Land and A. G. Doig, "An Automatic Method of Solving Discrete Programming Problems," Econometrica, vol. 28, no. 3, pp. 497-520, 1960.

[17] M. Nowak and W. Römisch, "Stochastic Lagrangian Relaxation Applied to Power Scheduling in a Hydro-Thermal System under Uncertainty," Annals of Operations Research, vol. 100, no. 1-4, pp. 251-272, Dec. 2000.

[18] R. Nürnberg and W. Römisch, “A Two-Stage Planning Model for Power Scheduling in a Hydro-Thermal System Under Uncertainty," Optimization and Engineering, vol. 3, no. 4, pp. 355-378, Dec. 2002.

[19] M. Tahanan, W. van Ackooij, A. Frangioni, and F. Lacalandra, "Large-scale Unit Commitment under uncertainty," 4ORQ J Oper Res, vol. 13, no. 2, pp. 115-171, Jan. 2015.

[20] W. van Ackooij, "A comparison of four approaches from stochastic programming for large-scale unit-commitment," EURO J Comput Optim, pp. 1-29, Sep. 2015.

[21] HydroByte, HydroData XP. http://www.hydrobyte.com.br/site, 2015.

[22] A. Frangioni, Generator of UC instances. http://www.di.unipi.it/optimize/Data/UC.html, 2008.

[23] A. Frangioni and C. Gentile, "Perspective cuts for a class of convex 0-1 mixed integer programs," Math. Program., vol. 106, no. 2, pp. 225-236, Jul. 2005.

[24] G. Morales-España, J. M. Latorre, and A. Ramos, "Tight and Compact MILP Formulation for the Thermal Unit Commitment Problem," Power Systems, IEEE Transactions on, vol. 28, no. 4, pp. 4897-4908, 2013.

[25] G. Morales-España, C. Gentile, and A. Ramos, "Tight MIP formulations of the power-based unit commitment problem," OR Spectrum, vol. 37, no. 4, pp. 929-950, May 2015.

[26] E. C. Finardi and E. L. da Silva, "Solving the hydro unit commitment problem via dual decomposition and sequential quadratic programming," Power Systems, IEEE Transactions on, vol. 21, no. 2, pp. 835-844, 2006.

[27] J. P. Vielma and G. L. Nemhauser, "Modeling disjunctive constraints with a logarithmic number of binary variables and constraints," Math. Program., vol. 128, no. 1-2, pp. 49-72, Jun. 2011

[28] A. L. Diniz, P. P. I. Esteves, and C. A. Sagastizabal, “A Mathematical Model for the Efficiency Curves of Hydroelectric units," in IEEE Power Engineering Society General Meeting, 2007, 2007, pp. 1-7.

[29] C. D'Ambrosio, A. Lodi, and S. Martello, "Piecewise linear approximation of functions of two variables in MILP models," Operations Research Letters, vol. 38, no. 1, pp. 39-46, Jan. 2010.

[30] B. Tong, Q. Zhai, and X. Guan, “An MILP Based Formulation for Short-Term Hydro Generation Scheduling With Analysis of the Linearization Effects on Solution Feasibility," IEEE Transactions on Power Systems, vol. 28, no. 4, pp. 3588-3599, Nov. 2013.

[31] Operador Nacional do Sistema Elétrico - ONS, "Diagrama Esquemático das Usinas Hidroelétricas do SIN,” 25-Aug-
2017. [Online]. Available:

http://www.ons.org.br/conheca_sistema/mapas_sin.aspx.

[32] O. Nilsson and D. Sjelvgren, "Variable splitting applied to modelling of start-up costs in short term hydro generation scheduling," Power Systems, IEEE Transactions on, vol. 12, no. 2, pp. 770-775, 1997.

[33] C. Lemaréchal, C. Sagastizábal, F. Pellegrino, and A. Renaud, "Bundle methods applied to the unit-commitment problem," in System Modelling and Optimization, J. Doležal and J. Fidler, Eds. Springer US, 1996, pp. 395-402.

[34] A. Frangioni, "Generalized Bundle Methods," SIAM Journal on Optimization, vol. 13, no. 1, pp. 117-156, 2002.

[35] E. D. Dolan and J. J. Moré, "Benchmarking optimization software with performance profiles," Math. Program., vol. 91, no. 2, pp. 201-213, Jan. 2002.

[36] A. Belloni, A. L. D. Lima, M. E. P. Maceira, and C. A. Sagastizábal, "Bundle Relaxation and Primal Recovery in Unit Commitment Problems. The Brazilian Case," Annals of Operations Research, vol. 120, no. 1-4, pp. 21-44, Apr. 2003.

[37] A. Daniilidis and C. Lemaréchal, "On a primal-proximal heuristic in discrete optimization," Math. Program., vol. 104, no. 1, pp. 105-128, Sep. 2005.

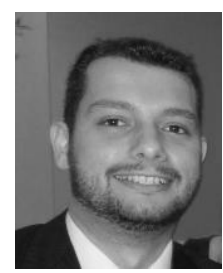

Murilo Reolon Scuzziato received the master degree and PhD in Electrical Engineering at Universidade Federal de Santa Catarina (UFSC) in 2011 and 2016, respectively. Since 2009, he has been developing research activities in electrical power systems planning, hydrothermal unit commitment and applied mathematical programming. He is now Assistant Professor at IFSC.

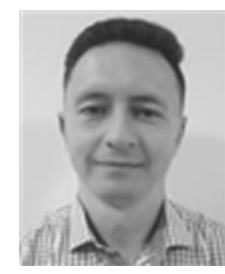

Erlon Cristian Finardi received the master and doctor degrees in Electrical Engineering from the UFSC, in 1999 and 2003, respectively, where he is now Associate Professor. His main research interest are hydrothermal power system planning, energy markets, smart grids and numerical optimization methods.

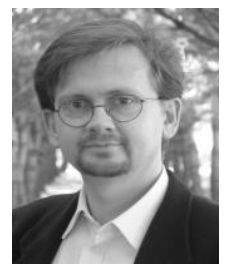

Antonio Frangioni graduated with honors in Computer Science from the University of Pisa, Italy, in 1992, got his Ph.D. in Computer Science from the same University in 1996, and spent all his career there, where he is now Full Professor since 2014. His main research interests are in models and algorithms for large-scale continuous and combinatorial optimization problems, using such techniques as decomposition algorithms, interior-point methods, reformulation techniques and network flow approaches, and applying them to diverse fields such as energy, telecommunications, logistics, and others. He is author and co-author of more than 50 journal articles, not counting several book chapters and conference presentations. 